\title{
L'enterrement chez les Mongols contemporains : le cercueil, la tombe et la yourte miniature du mort
}

The burial among contemporary Mongolians: the coffin, the grave and the miniature yurt of the dead person

\section{Sandrine Ruhlmann}

\section{(2) OpenEdition}

\section{Journals}

Édition électronique

URL : https://journals.openedition.org/emscat/1521

DOI : 10.4000/emscat.1521

ISSN : 2101-0013

Éditeur

Centre d'Etudes Mongoles \& Sibériennes / École Pratique des Hautes Études

Référence électronique

Sandrine Ruhlmann, «L'enterrement chez les Mongols contemporains : le cercueil, la tombe et la yourte miniature du mort », Études mongoles et sibériennes, centrasiatiques et tibétaines [En ligne], 40 | 2009, mis en ligne le 30 décembre 2009, consulté le 13 juillet 2021. URL : http://

journals.openedition.org/emscat/1521; DOI : https://doi.org/10.4000/emscat.1521

Ce document a été généré automatiquement le 13 juillet 2021.

(c) Tous droits réservés 


\title{
L'enterrement chez les Mongols contemporains : le cercueil, la tombe et la yourte miniature du mort
}

\author{
The burial among contemporary Mongolians: the coffin, the grave and the \\ miniature yurt of the dead person
}

Sandrine Ruhlmann

1 Au début de l'hiver 2000, un veuf a accepté que j'assiste à l'enterrement de son épouse et que je participe à toutes les étapes du rituel funéraire. Le rituel dure quarante-neuf jours divisés en séquences de sept jours, à partir du troisième jour après la mort idéalement fixé comme date d'enterrement. Ce veuf est l'oncle paternel aîné du maître du foyer chez qui j'ai élu résidence. Sa famille étendue vit à Öndörhaan, capitale de la province du Hentij au nord-est de la Mongolie. Depuis la mort de sa femme, il vit dans la maison en dur de sa fille aînée, mitoyenne de celle de sa fille cadette. Dans la même cour d'habitation, la défunte est isolée jusqu'à la mise en bière, dans la yourte où elle demeurait de son vivant avec son mari, parce que son corps constitue une "souillure " (buzar) majeure. Pour signaler la présence de la souillure que représentent la mort, le cadavre et l'idée même de la mort, la porte de la yourte est condamnée par une planche en bois, la cheminée est ôtée de son axe, le feu du fourneau n'est plus entretenu et l'ouverture supérieure de la yourte est entièrement obturée par son carré de feutre de fermeture. La yourte est momentanément aménagée en une yourte mortuaire, c'est-àdire en une yourte dont les éléments principaux sont inversés; elle est concrètement soumise à une fermeture, de manière à couper le défunt de l'extérieur ${ }^{1}$. À l'issue des quarante-neuf jours de deuil, la yourte mortuaire redeviendra une yourte d'habitation, où le veuf vivra seul.

2 Cette famille halh est de confession bouddhiste. Le jour suivant le décès, elle se rend au temple, situé dans le centre d'Öndörhaan, et consulte un lama qui indique comment bien procéder à chaque étape du rituel funéraire ${ }^{2}$. Souillée par la mort contagieuse, la 
famille restreinte, aidée de la famille étendue, s'évertue à bien enterrer la défunte pour favoriser le départ et le bon sort de son « âme » (süns).

Des personnes âgées énoncent encore parfois le souhait d'être, à leur mort, déposées à même le sol sur la terre de leurs ancêtres. La pratique de dépôt des morts dans la steppe remonte au $\mathrm{XVI}^{\mathrm{e}}$ siècle et a été promue par le clergé bouddhique. Mais les familles enterrent aujourd'hui leurs défunts conformément à la loi mise en place en 1955 par le régime communiste, qui recommande d'inhumer les corps, de préférence dans des cercueils 3 . Or ce mode d'inhumation n'est pas récent chez les peuples mongols, qui utilisaient des cercueils en bois depuis le $\mathrm{XVI}^{\mathrm{e}}$ siècle au moins pour enterrer les seigneurs mongols ${ }^{4}$ : leur dépouille était généralement installée dans un cercueil enfermé dans une structure en bois posée, par terre, au sommet des montagnes ${ }^{5}$.

Le cercueil n'est pas une simple boîte. Toutes les étapes techniques de construction, de décoration et d'enterrement du cercueil sont révélatrices du traitement de la " chair » (mah) en décomposition et des « os » (jas) qui se dissocient, et des croyances sur le sort post mortem de l'âme. À travers l'étude technique et ethnologique d'un objet, le cercueil, j'analyserai un type courant de pratique rituelle d'enterrement et la conception de l'âme après la mort répandu chez les Halh de Mongolie contemporaine, une république laïque où, après soixante-dix années d'athéisme communiste, la religion s'impose finalement dans les pratiques et à la conscience ${ }^{6}$.

\section{La construction et la décoration du cercueil en bois}

5 Le lendemain du décès, les gendres et autres parents masculins proches de la défunte se rendent au marché pour faire découper des planches de pin. Celles-ci serviront à construire le cercueil appelé « berceau » (ölgij) ou « boîte » (hajrcag). Les dimensions du corps de la défunte sont prises par la femme du neveu du veuf, couturière et menuisière de métier (chez qui je vis). Devant le portail de la cour d'habitation, les planches sont sciées sur mesure par les gendres de la défunte et un cousin du mari de la couturière. Le ponçage des planches est réalisé au centre de la cour d'habitation. Les planches doivent être assemblées et clouées avant le coucher du soleil, pour ne pas attirer les «mauvais esprits » (muu süns) $)^{7}$ qui rôdent la nuit. Le cercueil en bois a la forme d'un trapèze. La tête de la défunte reposera sur la base la plus large du trapèze; les pieds seront au niveau du sommet du trapèze. Durant la construction du cercueil, les visiteurs se succèdent pour saluer le veuf dans la maison de la fille aînée.

\section{Les couleurs internes cachées : le corps du mort sous le ciel et sur la terre}

\section{Le bleu du ciel}

6 Le soir, alors que le soleil est couché, le neveu du veuf, son cousin, deux neveux de la défunte et la fille aînée de la défunte, ainsi que la couturière-menuisière, installent deux tréteaux en bois dans la pièce de la maison en dur de la fille cadette de la défunte. Pendant la confection, les hommes et les femmes se concertent, à voix basse, quant à la manière de procéder, mais la couturière-menuisière a le dernier mot, parce qu'elle $a$ récemment participé à un enterrement à la capitale Ulaanbaatar, où les manières de procéder sont actualisées ${ }^{8}$. Elle sait avec précision comment les pompes funèbres 
décorent aujourd'hui les cercueils et comment les morts sont ensuite enterrés selon les directives du clergé bouddhique9.

7 Le « couvercle » (havhag) du cercueil est placé sur les tréteaux. L'ouvrage est commencé par l'intérieur du couvercle. Une fine couche de coton blanc recouvre toute la face interne grossièrement poncée. La couturière-menuisière sort d'un sac des pans de «tissus » (daavuu) en nylon: bleu, vert, rouge, noir et blanc. Les hommes tiennent la structure en bois et plantent les clous, pendant que les femmes maintiennent le revers du tissu vers l'intérieur du bois, pour une fixation esthétique. Chaque détail doit être soigné car "le cercueil doit être beau ", dit la couturière-menuisière dirigeant les opérations. Le tissu bleu recouvre le coton sur toute la surface interne du couvercle. Le visage de la défunte sera dirigé vers cette face «bleue » (höh), couleur indiquée par les lamas et adoptée sans difficulté par les familles puisqu'elle représente le « ciel » (tenger) des Mongols ${ }^{10}$. La défunte « regarde[ra] le ciel », me dit-on.

8 Au fur et à mesure que le tissu du ciel est fixé, les hommes et les femmes se déplacent autour du couvercle dans le sens inverse de circulation dans la yourte et de circumambulation autour des temples (autrement dit, dans le sens inverse des aiguilles d'une montre). La réalisation de "la décoration du couvercle est particulièrement importante, parce que c'est le ciel » explique la couturière-menuisière, qui découpe sept boules de coton blanc, de même taille, et les colle sur le tissu bleu, à l'emplacement de la tête du mort dans le cercueil. Ces boules de coton sont les «sept étoiles » (doloon od) ou les « sept divinités » (doloon burhan) représentant la Grande Ourse, et « le chiffre sept porte bonheur en Mongolie ", comme tout chiffre impair. La défunte "regarde les sept étoiles... et le ciel ». Le tissu bleu est placé à l'intérieur du couvercle pour que la défunte soit en contact direct avec le ciel et les divinités bouddhiques ${ }^{11}$. Les membres de la famille présents acquiescent, confirment chacun des propos de mon informatrice privilégiée, qui ne parle pas qu'en leur nom, mais pour tous les Mongols.

\section{Le vert de la terre et le territoire des ancêtres}

9 Dans l'autre pièce non communicante de la maison sont réunis le frère aîné et la fille cadette de la défunte, le veuf et des proches parents du veuf. Avec la même technique et une répartition similaire des tâches, ils décorent l'intérieur de la boîte du cercueil, préalablement couvert de coton blanc. Un tissu blanc est cloué sur les parois latérales et sur le fond, le fond étant ensuite recouvert de tissu vert.

10 Le vert n'étant traditionnellement pas une couleur proprement définie pour les Mongols ${ }^{12}$, la couturière explique sa présence dans la décoration du cercueil : le corps de la défunte va « reposer dans le cercueil sur la terre ». Le référent n'est pas la couleur verte de l'herbe, mais une notion de sol qui lui est associée. La couleur verte (nogoon) représente la «terre » (gazar), celle « des éleveurs nomades et du bétail », autrement dit le territoire sur lequel vit un foyer, une famille et les pâturages sur lesquels paissent ses troupeaux. Par «terre», les Mongols entendent aussi le territoire des ancêtres ${ }^{13}$. Beaucoup de Mongols situent leurs ancêtres sur une hauteur qui dépend de la division administrative de leur lieu de résidence ou de naissance ${ }^{14}$.

11 Le bleu du ciel et le vert de la terre associée au territoire des ancêtres sont les couleurs internes, non visibles, dites « cachées » (dald) du cercueil, choisies par les familles : elles sont en contact direct avec le corps du mort et évoquent la pratique ancienne de dépôt du cadavre dans la steppe. Si le bouddhisme impose une nouvelle couleur, le vert, 
originellement dénuée de valeur symbolique pour les Mongols, les familles se l'approprient et lui confèrent un sens, rejoignant des croyances populaires qui sont le fruit d'un entremêlement intéressé de chamanisme et de bouddhisme.

\section{Du blanc pour l'âme du mort}

Les parois internes de la boîte ont été parées de tissu blanc, alors que, selon les propos de la couturière-menuisière, la couleur blanche (cagaan) ne fait habituellement pas partie de la décoration du cercueil. Elle est ajoutée lorsque le cadavre est exceptionnellement enveloppé dans un linceul rouge, et non pas blanc, parce que le lama a déterminé la cause du décès comme étant accidentelle - c'est le cas de notre défunte -, et non pas naturelle.

Mon informatrice explique que le linceul blanc, à défaut, le tissu blanc placé sous le tissu vert, représente la peau blanche d'agneau anciennement installée, selon d'anciennes croyances chamaniques, sous le corps du défunt exposé dans la steppe ${ }^{15}$ dans tous les cas, une telle peau est toujours placée dans le fond de la fosse, sous le cercueil (cf. schéma 4).

Le tissu blanc est présent dans la composition décorative du cercueil pour que l'« âme de la défunte soit blanchie des péchés [qu'elle a commis de son vivant]». Ainsi, l'âme devient une "âme blanche » (cagaan setgel) ${ }^{16}$, la pureté étant incarnée par la couleur blanche "cagaan» représentant le caractère faste incarné par les laitages, les « aliments blancs » (cagaan idee) ${ }^{17}$.

15 La couleur blanche est donc ici présente à deux titres : elle représente l'ancien mode d'inhumation des morts dans la steppe, que beaucoup de Mongols considèrent comme étant le mode d'inhumation mongol traditionnel, ancestral, chamanique, et non imposé par le clergé bouddhique pour les hommes du commun, ainsi qu'une notion bouddhique de la pureté de l'âme.

L'intérieur du cercueil

la boîte

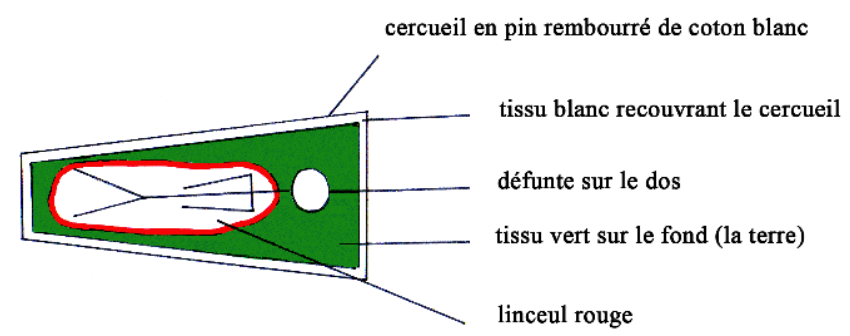

le couvercle

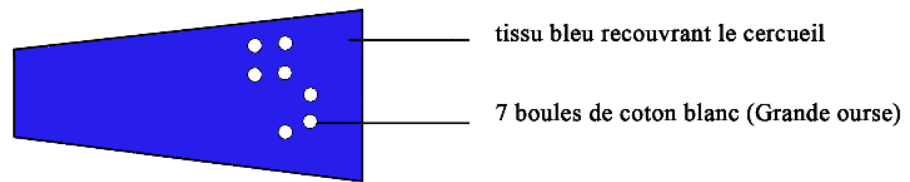



discours et les représentations de l'au-delà où l'âme attend de renaître. Le pays des âmes chamanique et le "pays des divinités" (burhany oron), sorte de paradis bouddhique, sont ici confondus, matérialisés et symbolisés par le ciel et le bleu, par le ciel bleu ${ }^{18}$.

\section{Les couleurs externes visibles : les couleurs étatiques du deuil} cercueil décoré, les hommes retournent la boîte et le couvercle et clouent les pans de tissu rouge, en sorte de recouvrir toute la surface extérieure du cercueil. Des bandes de tissu rouge et noir larges de dix, vingt et trente centimètres, sont découpées, plissées et alternées de façon à former «trois fleurs » (gurvan ceceg) noire et rouge. Sur le tissu rouge du couvercle, la grosse fleur est fixée à l'emplacement du visage de la défunte, la moyenne au niveau de son bassin, la petite au niveau de ses pieds. La couturière coud ensuite deux bandes de tissu noir de vingt centimètres de largeur, en diagonale des coins du cercueil donnant à l'emplacement de l'épaule droite et du pied gauche de la défunte. Enfin, elle confectionne des paires de lacets rouges et noirs, qui seront clouées sur les parois latérales extérieures de la boîte, de chaque côté d'un rectangle de tissu noir préalablement fixé sur le fond rouge.

La couturière explique que la couleur rouge représente la "tristesse " (uitgar) et la « dernière pensée » (süülijn sanaa) que la famille a pour le mort le jour de l'enterrement. Aujourd'hui, si aucun commentaire n'est fait sur la couleur noire prise isolément, il apparaît que, associée au rouge, elle symbolise le chagrin et qu'elle devient la couleur du deuil, notamment à Ulaanbaatar où, selon mon informatrice, les personnes riches revêtent un costume noir ou gris foncé pour se rendre à un enterrement. Quant à l'association du rouge de la tristesse et du noir du deuil russe, c'est à la fois, dit-elle, pour marquer le « respect » (hündlel) et signaler matériellement, extérieurement, que la famille est en deuil, le terme gašuudal « deuil » désignant également la tristesse. Cette association de couleurs est "politique ", "communiste", selon mon informatrice privilégiée. Caroline Humphrey (1999 : 66) précise que l'influence russe était très forte en Mongolie dans les années 1950-1960 et qu'à la capitale, la présence d'un mort et le deuil sont signalés sur la porte de l'appartement au moyen de bandes rouges et noires ${ }^{19}$. Le jour de l'enterrement, j'ai constaté que les membres du cortège funéraire arborent, en haut de leur bras gauche, un brassard fait de deux bandes, rouge et noire. Selon Humphrey, le brassard est une indication de deuil.

Les pleurs et la tristesse doivent traditionnellement être cachés, pour ne pas attirer l'attention des esprits néfastes et des âmes de morts errantes à la recherche d'un corps pour être nourries. C'est donc une couleur, en l'occurrence politique, et non religieuse, qui symbolise le chagrin de la perte d'un être cher - car même si l'âme renaît, il s'agit d'une autre personne.

La présence des couleurs emblématiques du socialisme soviétique, le rouge et le noir associés, montre explicitement que même l'athéisme soviétique ne peut se passer de supports matériels symboliques ou significatifs, ici d'ordre politique : sur ce point, il fonctionne à la manière d'une religion, alors même qu'il s'est donné pour vocation d'éradiquer toute forme de croyance et de pratique religieuse. Ces couleurs sont, non 
innocemment, les couleurs extérieures, visibles, du cercueil qui ne sont pas en contact direct avec le corps du défunt.

L'extérieur du cercueil

le couvercle

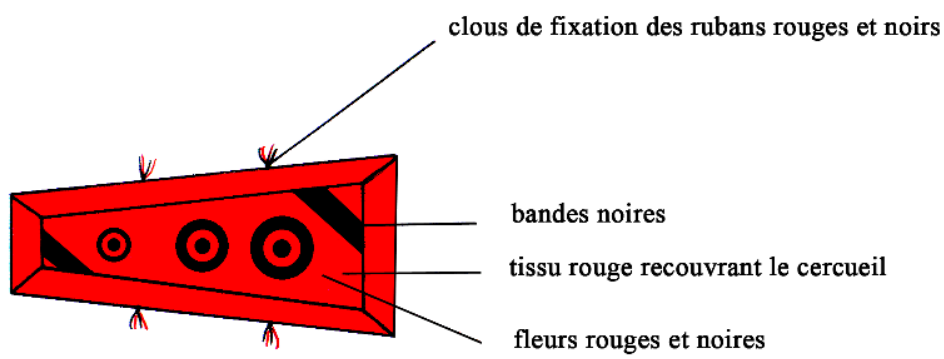

la boîte

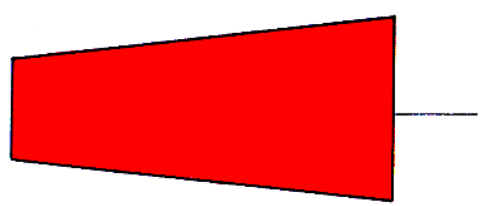

tissu rouge recouvrant le cercueil

les parois latérales

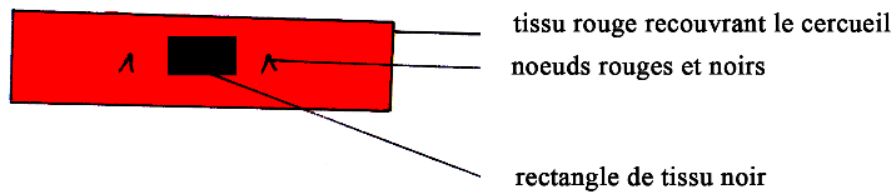

Schéma 2

21 Si l'influence soviétique est indéniable, de nouvelles influences, qualifiées pour certaines de traditions chamaniques, naissent des ouvrages publiés par des chercheurs mongols en sciences sociales. Ainsi, la pratique qui consiste à enrouler le cadavre dans un linceul blanc et à l'enterrer dans un cercueil dont l'intérieur est bleu et vert, a émergé suite à l'enterrement de l'auteur mongol Rinchen, en 1978, réalisé conformément aux pratiques décrites dans son livre (1958). Aucun texte politique ou religieux, aucune loi n'impose aux familles une quelconque décoration du cercueil, des couleurs à l'intérieur ou à l'extérieur. Pourtant, les familles prennent conseil auprès d'un lama pour bien enterrer les morts. Elles se font confirmer l'emplacement des sept étoiles et des trois fleurs. Elles placent, en contact direct avec le corps du défunt (partie non visible pour les vivants mais visible, pensent-elles, pour le mort), des couleurs qui revendiquent des croyances, des couleurs qui sont polysémiques et qui font sens, signifiant le dépôt du corps dans la steppe et la libération de l'âme qui quitte le corps pour poursuivre le cycle de la vie. Enfin, les couleurs qui sont visibles pour les vivants et non visibles pour le mort sont sans contact avec le mort et ces couleurs n'ont donc pas de rapport avec le traitement religieux de l'âme.

On ne peut pas expliquer pour l'instant comment l'influence russe de l'association du rouge et du noir en contexte funéraire continue de s'imposer aux familles, ces couleurs étant devenues les couleurs officielles nationales du deuil dans les années cinquante. Cependant, les familles savent parfaitement comment les assortir et les placer sur la face externe du cercueil. Il est certain qu'aujourd'hui ces couleurs n'ont aucune valeur 
religieuse et qu'elles ne relèvent d'aucune croyance relative à la mort et au sort de l'âme à la mort chez les Mongols - sinon pourquoi ne figurent-elles pas à l'intérieur du cercueil?

Dans les textes de lois, aucune précision n'est faite sur la manière de décorer le cercueil et de les enterrer. Dans les capitales provinciales comme dans les villages, le service administratif des pompes funèbres n'intervient pas sur les pratiques d'enterrement et rien n'oblige à enterrer les morts dans des cercueils dont les faces non apparentes sont recouvertes de bleu et de vert. Si le gouvernement encourage cependant financièrement de recouvrir les faces apparentes des couleurs officielles de deuil, le rouge et le noir - comme de bétonner les tombes, selon Grégory Delaplace (2007, p. 49, 78) -, rien n'indique d'apposer des fleurs rouges et noires ${ }^{20}$, ni des étoiles blanches sur fond bleu, à l'extérieur et à l'intérieur du couvercle. Il existe certes un côté administratif de l'enterrement des morts (l'enregistrement des morts), un côté religieux (la consultation d'un lama), mais l'enterrement est finalement laissé aux mains de la famille. La famille de «notre » défunte décore le cercueil comme ils le sont à la capitale, y compris par les pompes funèbres, et mon informatrice privilégiée sait justifier et expliquer l'utilisation symbolique de chacune des couleurs et celle, significative, des symboles.

\section{La mise en bière, le transport et l'enterrement du corps}

Les manières de porter le mort, de le déposer dans son cercueil, de transporter et de déposer le cercueil sur le sol, d'installer la tombe et d'enterrer le mort suivent, aujourd'hui, la logique de décoration du cercueil motivée à la fois par des croyances religieuses chamaniques et bouddhiques, des mesures politico-religieuses bouddhiques, ainsi qu'une idéologie politique hygiéniste et athéiste du traitement de la mort.

\section{Le port des os}

La mise en bière met un terme à la période d'isolement du cadavre. Le surlendemain de la construction du cercueil, le troisième jour après le décès, les "porteurs d'os " (jas barih hümüüs) ${ }^{21}$ portent le corps de la défunte, enveloppé nu dans son linceul, et le déposent allongé sur le dos dans la boîte du cercueil. Si l'expression de porteurs d'os a été conservée et désigne aujourd'hui les personnes qui portent le cercueil, elle désignait au XVI ${ }^{e}$ siècle le dépôt du corps dans la steppe. Les carnassiers dévoraient la chair exposée et, en brisant le squelette, ils libéraient l'âme logée dans les ${ }^{22}{ }^{22}$. Les "porteurs d'os", habits retroussés ou portés à l'envers, déplacent le cercueil dans le sens inverse de circulation dans la yourte, ordinairement néfaste (schéma 3), et ils le sortent de la yourte à l'envers, soit la tête et les épaules du mort avant ses pieds (schéma 4). 


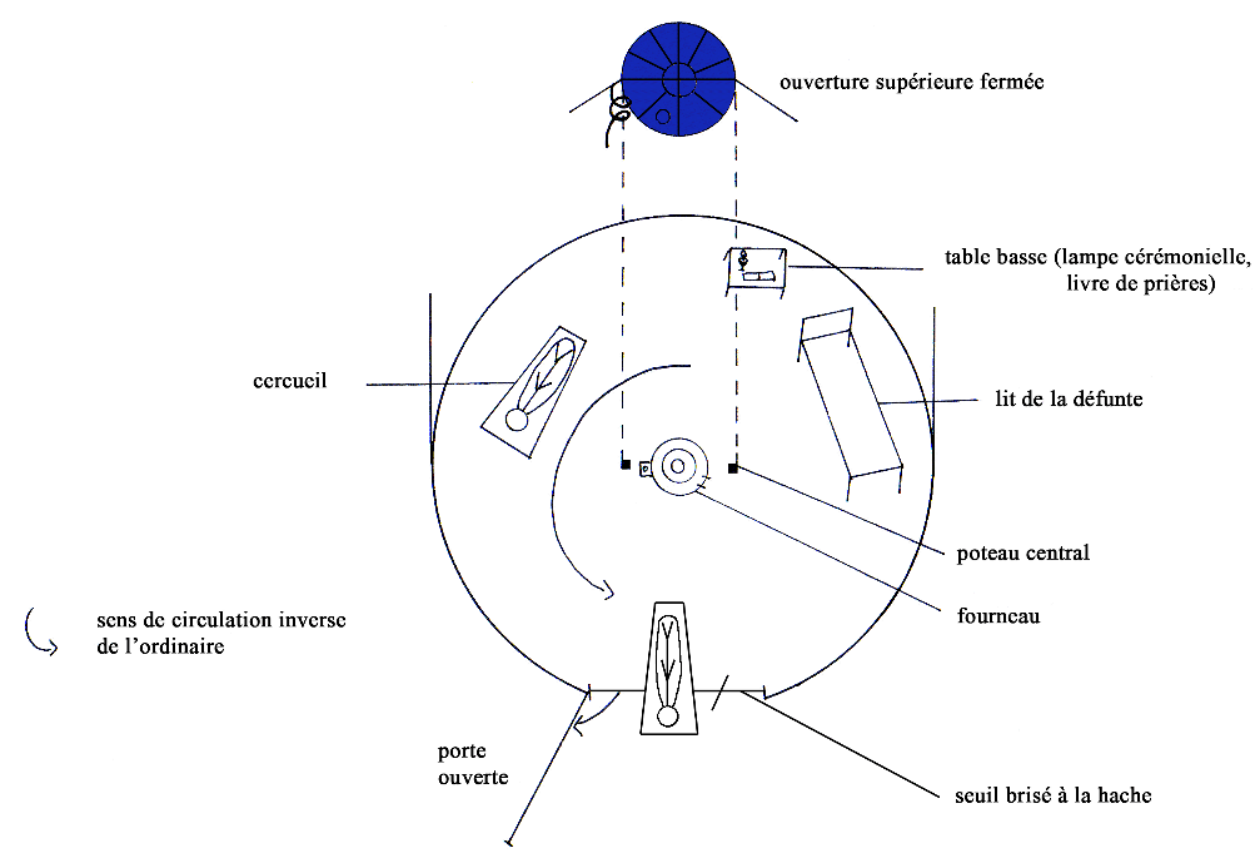

Schéma 3

Préparation de la fosse

brique de thé

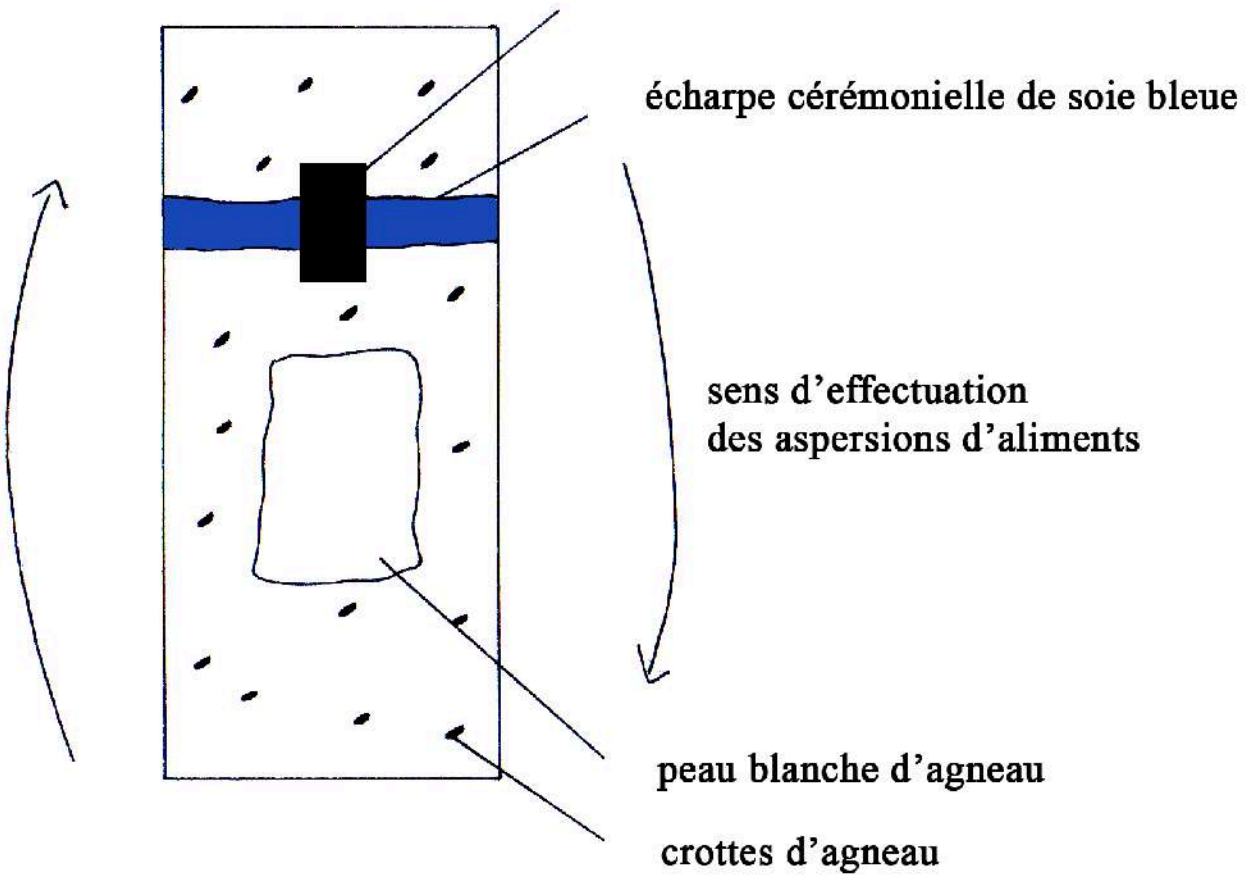

Schéma 4

Ils brisent ensuite le seuil de la yourte à la hache, tandis qu'il ne faut à l'ordinaire pas même le heurter pour ne pas fâcher les esprits censés y loger. Ces rites d'inversion d'actes quotidiens constituent une protection contre la souillure à laquelle les 
"porteurs d'os" sont directement exposés en portant le cadavre (Ruhlmann 2006, p. 458), et des inversions se retrouvent jusque dans les nourritures offertes au repas des funérailles (Ruhlmann 2009).

Avant le lever du soleil, le cercueil est placé à l'arrière d'un camion. Les membres du cortège funéraire s'assoient autour. La fille aînée porte le portrait funéraire de sa défunte mère, réalisé à partir d'une photo d'identité faite de son vivant ${ }^{23}$, à la hauteur de son visage dans le cercueil, ce jusqu'à la fin de la cérémonie d'enterrement. Une femme de la parenté de la défunte effectue une aspersion de thé au lait sur l'arrière du convoi funéraire qui sort de la cour d'habitation. Elle souhaite aux membres du cortège funéraire et au mort une route sans encombre dite «blanche » (cagaan zam). Au niveau $\mathrm{du}$ seuil de la cour d'habitation, le camion passe entre deux feux purificateurs ${ }^{24}$, allumés au moyen d'une braise du feu du foyer domestique - en l'occurrence, celui de la fille aînée de la défunte. Sur le chemin du cimetière, le chauffeur procède à des offrandes de graines de millet et de riz consacrées pour nourrir les âmes errantes de mort affamées et les mauvais esprits qui, dit-on, suivent le convoi funéraire.

\section{La tombe et le niveau de la terre} ars découvert - pour cela, le couvercle du cercueil est momentanément ouvert. La cérémonie de "faire ses adieux " (hagacal üjldeh) au mort est récente et serait une part affective imposée par les autorités mongoles dans les années 198027. Caroline Humphrey (1999, p. 73) interprète également cette affection comme le fruit d'une influence russe, le gouvernement mongol s'imprégnant alors du modèle soviétique. Les Mongols respectent cependant l'interdit traditionnel de pleurer son mort sous peine que son âme reste sur terre et nuise au bonheur de sa famille.

Le cimetière se situe en pleine nature, au pied d'une montagne. Cet emplacement est volontairement choisi: les Mongols ont un lien particulier avec les massifs montagneux, plus prononcé encore dans la région du Hentij aujourd'hui liée au souvenir de Gengis Khan, né sur la rive droite de l'Onon traversant le nord de la province ${ }^{25}$.

(hü̈r) déjà creusé26. Au fond de la fosse, ils déposent une «peau blanche d'agneau " (cagaan hurgany ar's) au niveau du bassin de la défunte dans le cercueil, et sèment des " crottes d'agneau » (hurgany argal) qui représentent le bétail prospère paissant sur les pâturages, explique mon informatrice. Une brique de thé chinois et une «écharpe cérémonielle [de soie bleue] » (hadag) y sont également installées, en offrande au mort, au niveau de la tête.

Les "porteurs d'os » déposent le cercueil à côté du trou sur un tapis vert synthétique rappelant le tissu vert du cercueil qui matérialise la «terre » (gazar). À cette étape du rituel, la famille est contrariée par l'écart entre les pratiques choisies et imposées, car le corps du mort devrait véritablement et non symboliquement reposer sous le ciel et sur la terre et non sous la terre comme l'impose la loi, me confie-t-on.

Le veuf prononce des vœux et adresse des éloges à sa défunte épouse, dont le visage est Il apparaît que le rituel funéraire est scandé, à des moments précis et significatifs, de rites bouddhiques ou chamaniques intercalés avec des manières de faire imputables à l'athéisme communiste, qui impose des sentiments affectifs plutôt que religieux. 
Adieux au mort

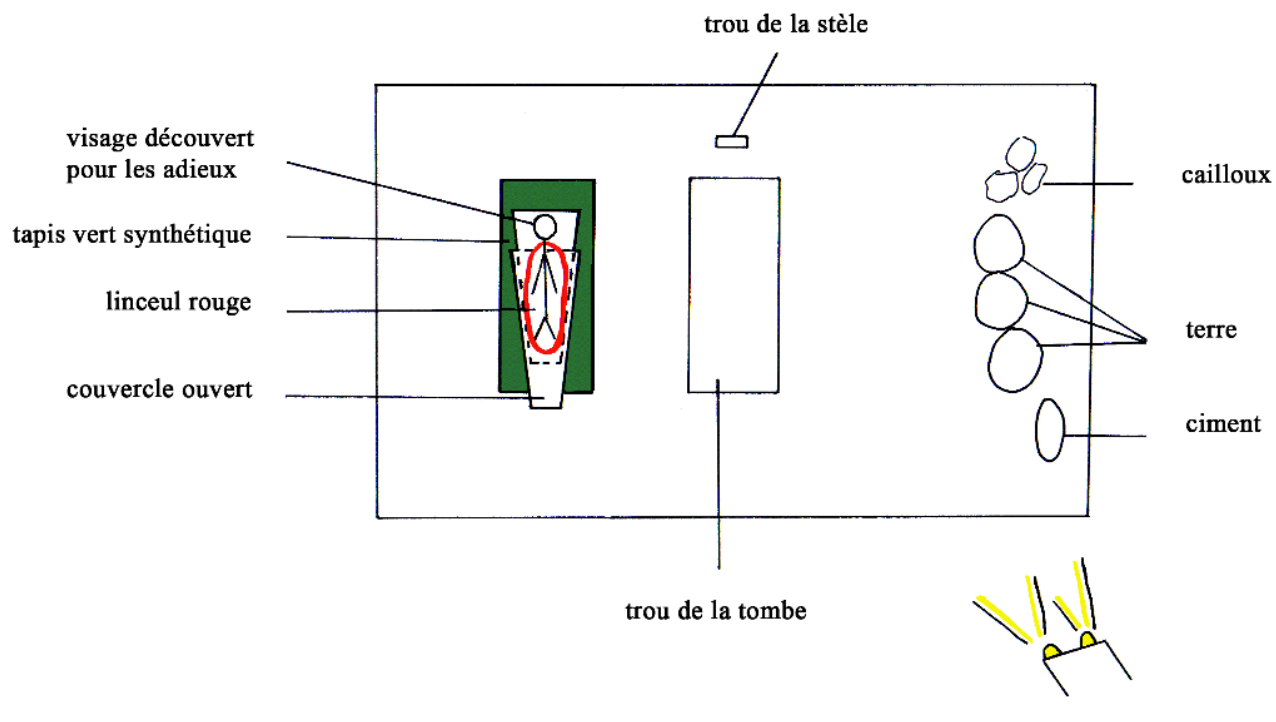

convoi funèbre

\section{Schéma 5} de graines consacrées de millet et de riz au-dessus de la fosse, dans le sens inverse des aiguilles d'une montre. Ils déposent ensuite le cercueil dans la fosse qu'ils remplissent de terre. Sur la tombe, ils forment un tumulus de terre renforcé de grosses pierres ${ }^{28}$. 


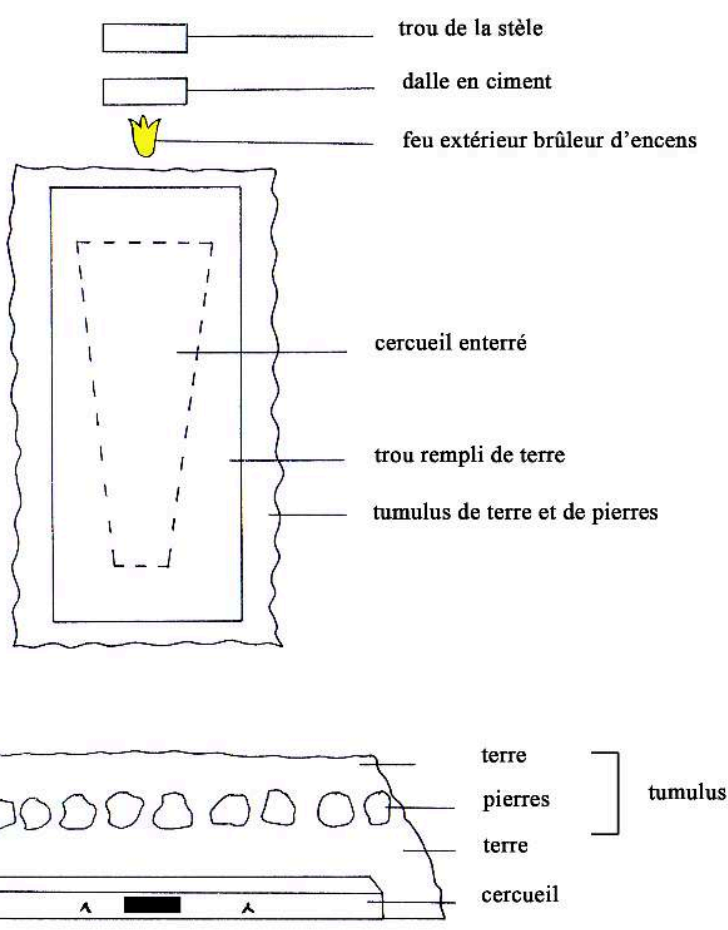

Schéma 6

S'ensuivent alors, par multiple de trois, des aspersions d'eau consacrée mêlée de lait, puis de graines consacrées, effectuées cette fois dans le sens coutumier et faste des aiguilles d'une montre - qui correspond au sens des circumambulations exécutées par séries de trois autour du monastère, en signe de bénédiction. Une stèle funéraire est cimentée au nord de la tombe, au niveau de la tête du mort dans le cercueil. Sur la stèle sont gravés l'initiale de son patronyme (prénom du père (ovog), comme à l'époque soviétique ; ovog est aujourd'hui de plus en plus un nom de clan en plus du patronyme), son prénom, l'année de sa naissance et de sa mort, ainsi que les trois flammes (représentant le passé, le présent et le futur), le soleil et la lune du sojombo ${ }^{29}$.

\section{Orientation du corps, de la tombe, du cimetière}

Dans la tombe, le mort a la tête au nord ${ }^{30}$, indiqué par les sept étoiles du cercueil formant la principale constellation de l'hémisphère nord du ciel, et les pieds au sud. Dans sa yourte, le maître de maison se tient assis dans la partie nord, devant l'autel domestique, le dos tourné à la montagne, et les jambes en direction de la porte d'entrée, soit vers le sud. Autrement dit, les morts se tiennent dans leur tombe comme les vivants se tiennent et se repèrent dans leur yourte : la tête au nord et les pieds au sud. 


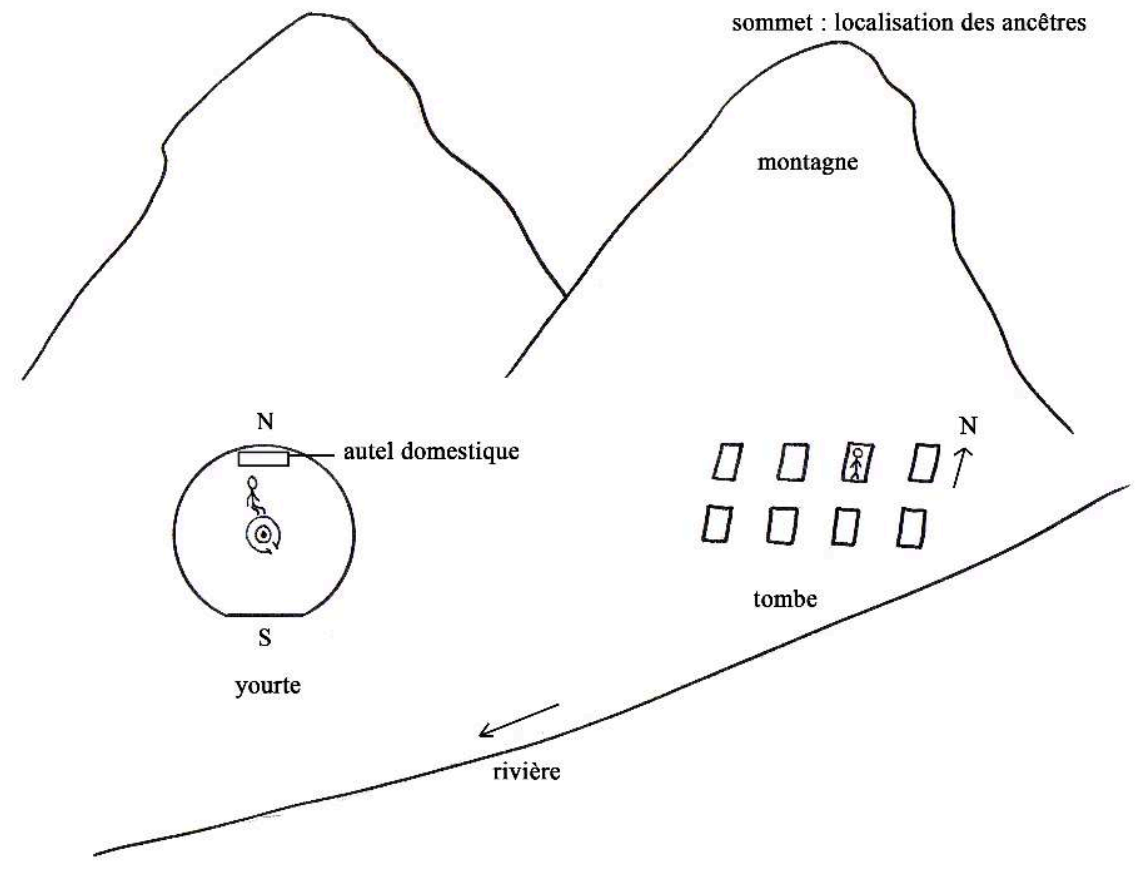

Schéma 7

Dans le paysage, le cimetière occupe un emplacement semblable à celui d'une yourte. L'un et l'autre se situent en bas d'une montagne, sur un terrain légèrement en pente descendante. Pour la yourte, la partie honorifique (hojmor) est idéalement orientée en direction $\mathrm{du}$ " nord» (hojno), physiquement vers le flanc de la montagne, tout comme l'arrière de la tombe (tête du mort). La partie «avant » (ömnö) de la yourte, indiquée par la porte d'entrée, est idéalement orientée en direction du «sud» (ömnö), physiquement sur une rivière située en contrebas, et ouvre sur le chemin pour quitter le campement ; la partie avant de la tombe (les pieds du mort) est orientée en direction du chemin emprunté par les vivants pour quitter le cimetière.

L'emplacement du cimetière, l'orientation des tombes parfaitement alignées dans le cimetière et l'orientation du corps dans les cercueils se font par rapport à l'espace naturel, une montagne, parce que son sommet est la localisation des ancêtres, ancêtres de la famille en particulier ou masse d'ancêtres. Au cimetière, les offrandes de nourritures se font au nord de la tombe, au niveau de la stèle funéraire, comme pour placer l'âme du défunt du côté des morts, le nord, le sud indiquant au contraire le chemin des vivants. Ainsi, dans la yourte, c'est sur l'autel domestique situé au nord que, d'ordinaire, la maitresse du foyer présente des nourritures en offrande aux morts récents, aux ancêtres et aux divinités bouddhiques.

Dans la tombe ou dans le cercueil, de nombreux éléments matériels et des couleurs rappellent le dépôt du corps dans la steppe : le vert du tapis synthétique, le bleu et le vert du cercueil, la peau blanche et les crottes d'agneau, ainsi que la terre jetée sur le cercueil au fond de la fosse jusqu'à former un monticule sur la tombe ${ }^{31}$. L'orientation du corps du mort et la représentation de la nature dans l'enterrement apparaissent comme étant essentiels pour l'âme du mort. 


\section{Le nourrissement de l'âme}

\section{La notion d'« âme » en Mongolie contemporaine}

39 En fonction des pouvoirs religieux et politiques gouvernant le pays sur les quatre derniers siècles, la notion d'âme a évolué, parallèlement aux modes d'inhumation, et s'est complexifiée à partir de la diffusion du bouddhisme réformé Gelugpa, à la fin du $\mathrm{XVI}^{\mathrm{e}}$ siècle. Ce bouddhisme est un mélange d'éléments doctrinaux, de croyances populaires tibétaines et de croyances chamaniques prébouddhiques ${ }^{32}$. Ainsi, les notions bouddhiques de péché, de jugement moral de l'âme et de karma individuel ${ }^{33}$ et les notions chamaniques d'«âme» (süns) et de "renaissance» (dahin törö-) se sont côtoyées dans la pensée des Mongols, faisant émerger de nouvelles conceptions de l'âme et de son sort après la mort, variables selon les régions. De nos jours, beaucoup de Mongols croient ainsi qu'à la mort, l'âme se déploie sous trois entités. Une âme (ou entité) reste près de la tombe jusqu'à ce que la chair se désagrège et que les «os » (jas) se dissocient - d'après des conceptions mongoles chamaniques anciennes. Une âme rôde autour de la yourte où séjournait le mort isolé, pendant la durée bouddhique du deuil de quarante-neuf jours, à l'issue duquel elle sera moralement jugée par le souverain Erlig des enfers bouddhiques. Enfin, une âme rejoint une sorte de pays des âmes, que les familles localisent de manière floue entre le ciel et la terre, ou, pour les plus fervents bouddhistes, le " pays des divinités » (burhany oron). Les familles ne savent pas dire si l'âme se divise en plusieurs entités, chacune occupant un lieu, ou si l'âme change de lieu suivant des étapes chronologiques, avant de renaître.

Les Mongols croient depuis toujours que le pays des âmes est un calque du monde des vivant $^{34}$ où l'âme séjourne jusqu'à renaître dans un descendant du mort ou, à défaut, dans un nouveau-né d'une autre lignée, respectant le principe chamanique de préservation de l'unité de vie que représente l'âme d'un homme. Aujourd'hui, ayant retenu la notion bouddhique populaire de «karma ${ }^{35}$, de nombreux Mongols font dépendre le sort de l'âme des morts des actions faites de leur vivant ${ }^{36}$. Dans le cas présent, si la famille fait un amalgame arrangeant entre les conceptions bouddhique et chamanique de "l'au-delà ", entre les idées de karma bouddhique et de renaissance propre à la société lignagère chamanique, elle s'applique à favoriser le départ de l'âme pour un au-delà clairement signifié par le ciel, soit la couleur bleue. Le bon sort de l'âme en jeu implique la famille jusqu'à la ruiner. Des funérailles coûtent entre 150000 et 300000 tougrig (de 125 à 250 euros), selon le type de service religieux, le nombre de repas offerts aux visiteurs, le prix du cercueil acheté ou fait maison et celui de la location du convoi funéraire. Elles représentent quinze à trente fois le salaire mensuel d'un foyer domestique ${ }^{37}$.

\section{Une yourte pour l'âme}

$41 \mathrm{Au}$ XIII ${ }^{\mathrm{e}}$ siècle, de grands seigneurs étaient ensevelis dans une de leurs demeures. Ils étaient assis devant une table, sur laquelle étaient présentés un plat de viande et du lait de jument fermenté. Un cheval était enterré en offrande au mort dans la fosse ${ }^{38}$ et un autre cheval était ensuite sacrifié en offrande aux mânes des ancêtres ${ }^{39}$. Il reste aujourd'hui, de la pratique récupérée de nourrissement du mort, le dépôt d'une "yourte» (ger) réalisée en miniature, telle une miniaturisation de la scène 
d'ensevelissement du mort nourri dans sa demeure. Selon certains Mongols, la pratique de dépôt d'une petite yourte sur la tombe est une récupération de croyances chamaniques ${ }^{40}$ pour "nourrir le mort », alors qu'elle a semble-t-il émergé dans les années 1980, sous l'impulsion du gouvernement communiste.

La yourte miniature est enfoncée dans une dalle en ciment, entre la stèle funéraire et la tombe. Fabriquée la veille à partir d'une boîte de thé ronde en fer et de fils de fer par le beau-fils de la fille aînée de la morte, cette yourte miniature est l'exacte réplique de la yourte et de sa structure architecturale.

La yourte miniature et son feu

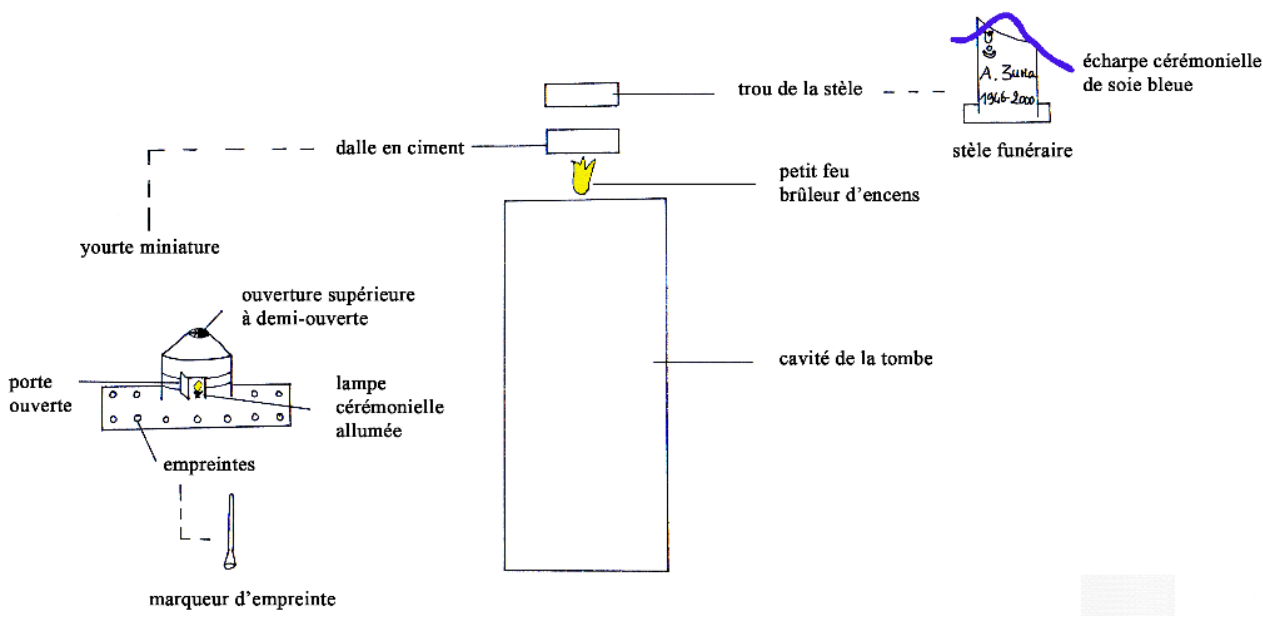

Schéma 8

La porte et le toit sont ouverts. Une lampe cérémonielle est allumée et placée à l'intérieur. Comme les maîtresses de foyer sont chargées de veiller sur le feu protecteur $\mathrm{du}$ foyer domestique, les membres féminins du cortège funéraire veillent le petit feu qu'elles ont allumé à l'extérieur de la petite yourte et l'entretiennent pendant la cérémonie d'enterrement. Cette yourte miniature est semblable à une yourte ordinaire - elle ne ressemble pas à la yourte mortuaire dans laquelle est isolé le cadavre avant d'être mis en bière et enterré. C'est dans cette yourte miniature que l'âme du mort est invitée à séjourner - des trois entités, elle est celle qui reste près de la tombe.

En contexte pastoral et rural bouddhisé, les familles ont une conception matérialiste de l'au-delà. Elles pensent, selon des croyances antérieures au bouddhisme, que les besoins des morts dans l'au-delà sont similaires à ceux des vivants ${ }^{41}$. Elles offrent donc à leurs morts (dans/sur la tombe) du thé (repas le plus élémentaire et offrande minimale d'hospitalité), du combustible (crottes d'agneau), du bétail (peau d'agneau), ainsi qu'une maison (yourte miniature) équipée d'une bougie (pour s'éclairer) et d'un feu (pour se chauffer et faire cuire des aliments). Les familles disent que le mort pourra " faire un bon feu, manger des raviolis, boire du thé, prier dieu et dormir avec un oreiller ». La mention des raviolis n'est pas insignifiante: ce sont des nourritures préparées pour être offertes aux visiteurs en situation d'hospitalité ou de fête. Telle qu'elle est présentée, la yourte miniature indique que l'âme logera dans un espace domestique, au sein duquel le mort conservera son statut et les rapports sociaux entretenus de son vivant. 
Si le mort est pourvu dans sa tombe de tout ce dont il a besoin pour vivre dans l'audelà, sa famille ne lui offre plus sur le lieu d'inhumation de la viande et des laitages comme cela se pratiquait jusqu'au $\mathrm{XVI}^{e}$ siècle, avant l'interdit bouddhique de sacrifice animal en association avec la mort d'un humain (Ruhlmann 2009).

\section{Une vie sociale dans l'au-delà}

Les vivants prennent une précaution ultime pour que l'âme ne souhaite pas rester icibas les tourmenter. Au moyen d'un suvraga ${ }^{42}$, un des membres du cortège funéraire réalise onze marques circulaires dans le ciment encore frais de la dalle, au niveau du seuil de la yourte miniature. Les marques délimitent en quelque sorte une frontière entre la vie de l'âme dans l'au-delà et le monde des vivants, donc entre la mort et la vie. Les onze marques sont faites «à la mémoire du défunt " pour qu'il « ne revienne pas à la maison ", explique mon informatrice. Ces marques sont une matérialisation des empreintes des pas des visiteurs faites pour l'apaiser et lui assurer qu'il recevra des visiteurs dans l'au-delà ${ }^{43}$.

Pour clore la cérémonie d'enterrement, tous les membres du cortège funéraire effectuent une dernière série de trois offrandes de graines consacrées sur la tombe, pour nourrir l'âme de la défunte et l'inviter à rester près de la tombe ${ }^{44}$. Les femmes s'assurent que le feu est encore allumé et que la fumée de l'encens qui y brûle « appelle l'âme [du mort] » à entrer dans la yourte miniature ${ }^{45}$. Hommes et femmes du cortège quittent le cimetière la conscience tranquille et ne se retournent plus en direction de la tombe pour ne pas inciter l'âme de leur défunte à les suivre.

\section{Un portrait photographique pour nourrir l'âme}

La famille dote l'âme d'une yourte où elle pourra se nourrir dans l'au-delà, mais elle doit également la nourrir d'une manière interposée ici-bas, pour éviter de la fâcher et ne pas l'inciter à rôder sur terre parmi les vivants. L'âme est donc nourrie sous la yourte où sa famille offre le repas des funérailles et les repas funéraires de commémoration ${ }^{46}$.

9 Seul le portrait photographique est rapporté du lieu d'enterrement à la maison. Il est une représentation matérielle de l'âme de la défunte. L'écharpe cérémonielle de soie bleue, qui ornait le portrait depuis la mise en bière, a été déposée sur la stèle funéraire à la fin de cérémonie d'enterrement, parce qu'elle renferme le dernier souffle de la défunte, dit-on. C'est par l'intermédiaire du portrait photographique que les familles nourrissent l'âme. Après trois ans, plus aucune nourriture n'est généralement déposée devant ce support d'âme, car les familles pensent que l'âme a rejoint l'au-delà, pour ensuite soit se loger dans le corps d'un descendant, s'inscrivant dans le cycle des renaissances, soit rester parmi les mânes d'ancêtres.

Dans la yourte des funérailles, anciennement yourte mortuaire, où se tient le repas des funérailles au retour des membres du cortège funéraire, le portrait est placé sur l'autel funéraire, dans la partie nord honorifique. La morte ne mange pas à la table des vivants, mais à la table des non-vivants: des êtres surnaturels, les divinités, représentées par des images, et des êtres anciennement décédés, les ancêtres de la famille, représentés par le portrait photographique de la défunte parce que son âme les rejoindra, comme le pensent certaines de ses parentes. Les Mongols disent ainsi que le 
mort est "devenu divinité » "burhan bolson", quand ils veulent signifier qu'il est décédé pendant la période de deuil où il est tabou de parler de la mort et du mort comme de prononcer les mots les désignant.

51 Des familles craignent que l'âme censée rester près de la tombe n'ait suivi le cortège funéraire jusqu'à la maison - sa maison de son vivant. Elles déposent donc à l'extérieur de la yourte des funérailles, au niveau du seuil, une assiette remplie des mêmes nourritures que celles qui sont offertes au portrait photographique à l'intérieur de la yourte, sur l'autel funéraire. Ainsi bien nourrie, l'entité rôdant peut être tentée de rester à leurs côtés, mais les familles sont confiantes, car les nourritures blanches offertes apaisent toujours les âmes et les esprits, qu'ils soient bons ou mauvais ${ }^{47}$.

\section{Conclusion}

Le cercueil, un objet que l'on croirait dénué d'intérêt, porte en lui les marques des évolutions historico-politiques des modes d'inhumation des morts en Mongolie, depuis le XIII ${ }^{\mathrm{e}}$ siècle au moins jusqu'à nos jours. Par un jeu de couleurs, de symboles et de signes dans ou sur le cercueil, mais aussi à l'extérieur de la tombe, les Mongols contemporains renouent avec des pratiques funéraires et des notions de l'âme bouddhiques, que l'athéisme communiste avait bannies, ainsi qu'avec des pratiques et des croyances chamaniques relatives à la mort, somme toute revisitées ou réinventées, c'est-à-dire avec un traitement particulier de la chair et des os, du corps et de l'âme, insufflées par des nouvelles formes de chamanismes qui apparaissent depuis une décennie dans des centres spécialisés à Ulaanbaatar. Le tout s'harmonisant sur un fond de politique athéiste qui se pérennise tout en établissant, à partir des années 1990, une tolérance religieuse et une démocratie républicaine. En fait, les pratiques et les croyances sur la mort, et en premier lieu le cercueil, renferment les stigmates des répressions religieuses et politiques successives : tout d'abord du clergé bouddhique sur la société mongole chamanique, puis, entre 1920 et 1980, du gouvernement communiste antireligieux sur la société mongole bouddhisée.

L'ironie du sort veut non seulement que le régime actuel, athée, fasse renouer le peuple avec la religion, mais qu'il annule les effets des répressions du chamanisme par le clergé bouddhique. Les lois de l'époque communiste athéiste ont abouti à ce que le peuple enterre aujourd'hui ses morts comme les empereurs chamanistes convertis au bouddhisme étaient enterrés (dans des cercueils enterrés dans des tombes constituant des cimetières). D'une part, les lois areligieuses et hygiénistes promulguées depuis la fin du régime communiste réactualisent un mode d'inhumation que le clergé bouddhique avait réservé aux nobles, alors que le gouvernement pensait combattre les pratiques et croyances bouddhiques d'inhumation. D'autre part, le régime actuel donne l'occasion aux Mongols d'être enterrés comme leurs empereurs gengiskhanides qu'ils considèrent comme étant leurs ancêtres directes, à l'encontre des intentions initiales du clergé bouddhique qui avait, au XVI siècle, imposé des modes d'inhumation différents pour les hommes du commun et les aristocrates, dans l'intention de couper les Mongols de leurs ancêtres et de briser l'organisation clanique de la société. En outre, il semble qu'il y ait aujourd'hui une confusion des modes d'inhumation et des croyances leur correspondant, notamment chez les personnes âgées qui souhaitent que leur corps soit exposé à la mode bouddhique des hommes du commun, pensant que ce mode d'inhumation relève d'une tradition chamanique prébouddhique. 
54 Auparavant, l'âme était libérée lorsque les os du corps exposé étaient brisés par un animal sauvage carnivore. Aujourd'hui, l'âme est considérée comme dissociée du corps enterré, alors que les os du squelette restent intacts. C'est pourquoi, de nos jours, les traitements rituels de l'âme ne correspondent plus de la même manière aux traitements rituels du corps : les familles réécrivent le scénario du rituel funéraire et consacrent à différentes étapes rituelles des symboles et des rites qui servent les notions mongoles contemporaines de la mort, de l'âme et de la renaissance. De nouveaux objets, la yourte miniature et le portrait photographique du défunt, sont investis des croyances religieuses essentielles liées à la mort : la renaissance de l'âme et la mémoire du mort. L'étude du cercueil, avec ses couleurs cachées et apparentes, permet de comprendre que la renaissance de l'âme n'est plus liée à la dispersion des os et ni empêchée par l'enterrement du corps. Si les néochamanes de la capitale en sont contrariés, les familles, à la fois bouddhistes, chamanistes, fengshuistes, républicaines, et capitalistes, semblent s'en accommoder parfaitement. Malgré une reviviscence des traditions religieuses mongoles d'avant la révolution de 1921 et la libéralisation du pays, les Mongols ne remettent pas en cause le mode communiste d'enterrement. Ils préfèrent composer, combiner, bricoler, pour servir les intérêts de leurs morts dans une société en mutation accélérée.

\section{BIBLIOGRAPHIE}

Ar'jasüren, ̌̌. et Njambuu, $\mathrm{H}$.

1992 Mongol jos zanšlyn ih tajlbar tol' [Grand dictionnaire des coutumes mongoles], tome 1.

(Ulaanbaatar, Hüühdijn Hevlelijn Gazar).

Badamhatan, S.

1987 BNMAU-yn ugsaatny züj.1 bot'[Ethnologie de la République populaire de Mongolie. $1^{\text {er }}$ tome],

(Ulaanbaatar).

Batu, Jirigala and Yang, Haiying

2005 Aerzhai shiku - Chengjisi han de fojiao jiniantang xingshuai shi - Arjai Grotto - The rise and Fall of a Buddhist Memorial for Chinggis Khan, Tokyo : Fukyosha Publishing. 186p.

Beffa, Marie-Lise et Hamayon, Roberte

1983 Les catégories mongoles de l'espace, Études mongoles et sibériennes 14, pp. 81-111.

Cevel, Ja.

1959 Mongolčuudyn erhemledeg öngö [Les couleurs préférées des Mongols], Studia ethnographica I.6 .

(Ulaanbaatar), pp. 3-5.

Chabros, Krystyna

1992 Beckoning fortune : a study of the Mongol dalaya ritual, (Otto Harrassowitz, Wiesbaden).

Delaplace, Grégory

2007 L'invention des morts en Mongolie contemporaine. Sépultures, fantômes, photographie. (Thèse de doctorat, EPHE, Paris). 381p. 
2008 L'invention des morts. Sépultures, fantômes et photographieen Mongolie contemporaine. Nord-Asie 1, CEMS, EPHE, 374p.

Delaplace, Grégory et Micoud, Vincent

2007 Les usages sociaux de la photographie en Mongolie. Question de méthode.

ethnographies.org 13, en ligne sur le site www.ethnographiques.org.

Even, Marie-Dominique

1999 L'au-delà dans les représentations religieuses des Mongols, in P. Servais (éd.), La mort et l'audelà : Une rencontre de l'Orient et de l'Occident, (Louvain-la-Neuve, Academia Bruylant), pp. 149-196.

Hamayon, Roberte

1978 Des fards, des mœurs et des couleurs. Étude d'ethno-linguistique mongole. in S. Tornay (éd.), Voir et nommer les couleurs, (Nanterre, Laboratoire d'ethnologie et de sociologie comparative), pp. 207-247.

Humphrey, Caroline

1999 Rituals of death in Mongolia : their implications for understanding the mutual constitution of persons and objects and certain concepts of property, Inner asia I.1., pp. 59-86.

Krueger, John R.

1965 The Altan Saba (The Golden Vessel): A Mongolian Lamaist Burial Manual, Monumenta Serica XXIV, pp. 207-272

Ligeti, Louis

1973 Le sacrifice offert aux ancêtres dans l'Histoire secrète, Acta Orientalia Academiae Scientiarum Hungaricae XXVII (2), pp. 145-161.

Mauss, Marcel

1899 Rites funéraires en Chine, Année sociologique 2, pp. 221-226.

Merli, Laetitia

2004 De l'ombre à la lumière, de l'individu à la nation : renouveau chamaniste en Mongolie postcommuniste. (Thèse de doctorat, EHESS, Paris).

Mostaert, Antoine

1968 [Peking, 1941-1944]Le Dictionnaire Ordos, (CICM), Johnson Reprint Corporation, New-York ( $1^{\text {ère }}$ édition par la Catholic University de Pékin).

Plan Carpin, Jean de

1965 Histoire des Mongols, traduit et annoté par J. Becquet et L. Hambis. (Adrien-Maisonneuve, Paris).

Pop, Rodica

2002 De la difficulté terminologique du clan chez les Mongols, Studia asiatica III, 1-2, pp. 59-73.

Pürev, Otgony et Pürvee, Gurbadaryn

2007 Mongolian Shamanism : revised and updated fifth edition, I et II, 322p.

Rinchen, B.

1958 Iz Našego Kul'turnogo Naslediya. (Ulsyn Khevlel, Ulaanbaatar).

Ruhlmann, Sandrine

2006 Le partage des prémices et du fond de la marmite. Essai d'anthropologie des pratiques alimentaires chez les Mongols Xalx. (Thèse de doctorat, EHESS, Paris). 574p.

2008a Les pratiques alimentaires funéraires chez les Mongols Xalx. Purifications, offrandes et repas. In La mort en montagne - Perceptions, représentations, rituels, sous la direction de G. Boëtsch, M. Signoli, S. Tzortzis, Editions des Hautes-Alpes (souspresse). 
$2008 \mathrm{~b}$ « Une curieuse pâtisserie en forme de semelle : le nouveau support de bonheur des mongols halh bouddhisés / An odd soleshoe-shaped pastry: the new support of happiness of Buddhist halh Mongolians ", Anthropology of Food, SO, Varia, le numéro permanent, [en ligne le 15 décembre 2008].

2009 Une soupe peu ordinaire. Analyse du repas des funérailles chez les Mongols, Techniques \& Culture 51, pp. 40-60.

\section{NOTES}

1. Dans certaines régions, le corps du défunt est sorti par une ouverture pratiquée dans le mur nord de la yourte (Humphrey 1999, p. 72; Even 1999, pp. 188-189). Le défunt est alors installé dans une yourte provisoire, spécialement montée pour l'occasion.

2. Pour les étapes préliminaires de l'enterrement, où la famille invite un lama astrologue à lire les prières du livre appelé "Altan sav » "boîte dorée » (Krueger 1965) et à désigner au cours du rituel d'«ouvrir la boite dorée" (altan sav neeh) les personnes autorisées à confectionner le cercueil, à porter le corps du défunt et à participer à la cérémonie d'enterrement et détermine la date de l'enterrement, voir Ruhlmann 2006, p. 523.

3. Si l'arrêté de 1955 oblige d'enterrer les corps dans des cimetières, l'utilisation du cercueil est déclarée facultative dans l'arrêté de 1956, pour être abrogée en 1972 (Delaplace 2007, p. 48), en réponse à la réticence des Mongols, pas tant à enterrer leurs morts, mais à les enterrer dans une boîte empêchant le contact du corps avec la terre, avec la nature.

4. Delaplace 2007, p. 141, Even 1999, p. 168. Pour une représentation en peinture de cercueil en bois dans une grotte d'Arjai en territoire Ordos, datant du XVI siècle, voir Batu Jirigala et Yang Haiying 2005, p. 186 (grotte 31, mur de droite). Une autre représentation figure dans le manuscrit de Walther Heissig (1989, p. 204). Je remercie Isabelle Charleux pour ces précieuses informations.

5. Bien que tenu secret, l'emplacement était veillé pour éviter les pillages des sépultures (Plan Carpin 1965, pp. 41-43).

6. À partir des années trente, le gouvernement communiste a supprimé toute forme de pratique chamanique et éradiqué les chamanes; il a également interdit les pratiques bouddhiques, mais il n'est pas parvenu à déraciner le clergé bouddhique, fortement implanté depuis plusieurs siècles.

7. L'expression englobe les âmes de morts errantes et les esprits néfastes ou fâchés par les maladresses et les mauvaises actions des vivants.

8. Toutes les parentes proches et éloignées d'un défunt doivent aider ses filles (à défaut, ses belles-filles ou ses soeurs) à préparer les repas funéraires et à recevoir les visiteurs présentant leurs condoléances à la famille. Cette femme en particulier, parce qu'elle est menuisière et couturière de métier, et parce qu'elle sait, pour l'avoir observé et pour y avoir participé, comment se déroule un enterrement suivant toutes ses séquences rituelles, et comment, de surcroît, on prépare le cercueil, depuis l'assemblage des planches jusqu'à sa décoration. Elle en supervise donc chacune des étapes, avec une autorité que ses "aînés " (ah) - les hommes - ne discutent pas.

9. Ces dernières dépendent notamment de la cause du décès, qu'un lama, désigné par le monastère, détermine en s'appuyant sur un manuel astrologique bouddhique, l'Altan sav (Krueger 1965), voir note 2 .

10. «L'éternel Ciel bleu » (Jean de Plan Carpin 1965, p. 142 note 13), auquel les Mongols n'ont cependant jamais rendu de culte.

11. Une flèche blanche pointant en direction des pieds peut remplacer les sept étoiles (Pürev et Pürvee 2007). Elle serait une récupération des traditions chamaniques selon lesquelles les morts montaient au ciel en laissant derrière eux une traînée blanche (signifiant qu'ils ont quitté leur famille sans laisser de souffrance) et renaissaient. 
12. Selon Hamayon (1978), l'apparition d'un terme propre pour la couleur verte, autrefois non distinguée de la couleur bleue, est relativement récente et présente surtout dans les milieux fortement bouddhisés. Les Mongols utilisent généralement le terme nogoon « vert » pour les verts artificiels et le terme höh « bleu » pour les verts naturels.

13. Des familles ont aujourd'hui tendance à fondre les ancêtres de leur famille et les ancêtres gengiskhanides dans un même ensemble formant une masse d'ancêtres. D'autres Mongols identifient les lignées princières de Gengis Khan et de ses descendants comme étant leurs ancêtres et s'approprient simultanément les ancêtres mythiques de Gengis Khan, le Loup Bleu et la Biche Fauve, situés dans la montagne Burhan Haldun des monts Hentij.

14. En général, avant le mariage, la résidence du couple est aujourd'hui matrilocale. Après le mariage, elle est patrilocale jusqu'à ce que le couple s'installe dans une «nouvelle yourte » (šine ger), suivant le principe de néolocalité.

15. Au temps du dépôt du corps dans la steppe, la peau déposée sur le sol était recouverte de soie verte (Badamhatan 1987, p. 302). Le tissu vert du cercueil serait-il une réplique de cette soie verte?

16. Selon mon information, les Mongols emploient indifféremment les termes süns ou setgel pour désigner l'âme du vivant, tandis que l'âme du mort se dit toujours "süns ». S'agissant des composantes liées à la notion d'âme, je laisse de côté la « force vitale » (süld).

17. Sur le symbolisme des couleurs chez les peuples mongols, voir Cevel 1959 et Hamayon 1978.

18. Les âmes en attente de renaître sont comme stockées en un lieu que les familles ne savent pas localiser. Depuis que la notion de paradis bouddhique est répandue, le pays des âmes est explicitement situé dans le ciel, sans davantage de précision.

19. Chez les Ordos, un tissu rouge permet d'interdire l'accès à une yourte quand une femme a accouché, quand un malade est alité ou qu'une personne est décédée (Antoine Mostaert 1968 [1941-1944], p. 657a, 699b, 700a). Cette pratique de marquage existe aussi chez les Halhs, selon les maîtresses des foyers chez qui j'ai vécu.

20. Les bureaux d'administration funéraire peuvent procurer aux fabricants de cercueil ou directement aux familles du tissu noir et rouge, donc du tissu des seules couleurs officielles, politiques, du deuil (Delaplace 2007, p. 49). Pour plus de détails sur les lois mongoles concernant l'enterrement dans des cercueils, voir Delaplace 2007.

21. Les « porteurs d'os » sont les parents masculins du veuf et de la défunte que le lama, consulté à la suite du décès, désigne en fonction de leur signe astrologique. Ils mettent en bière le corps, transportent le cercueil et le déposent dans la tombe. Ils dirigent les membres du convoi funéraire et sont chargés du bon déroulement de la cérémonie funéraire au cimetière.

22. Pour certains Mongols, elle est logée à la mort dans l'os atlas, la première vertèbre cervicale qui, supportant tout le poids de la tête, se briserait facilement (Ruhlmann 2006, p. 312). Des Mongols croient que l'âme est véritablement libérée, "sortie du corps ", lorsque l'os atlas se rompt, un ou trois ans après la mort. Cette croyance rejoint d'anciennes croyances chamaniques, selon lesquelles la désintégration des chairs et des os ne suffit pas à libérer l'âme (Even 1999, p. 186).

23. Sur ce point précis, lire la thèse de Grégory Delaplace 2007, pp. 307-356 et son article écrit avec Vincent Micoud 2007.

24. Au XIII ${ }^{\mathrm{e}}$ siècle déjà, il fallait brûler toutes les affaires personnelles d'un mort dans un grand feu pour éviter la contagion de la mort (Plan Carpin 1965, p. 37, 40, 43). Agent de chauffage, d'éclairage et de cuisson, habité par un esprit-maître protecteur du foyer domestique, le feu est aussi un agent de purification.

25. Voir Ruhlmann 2008a.

26. Généralement, un lama désigne l'emplacement de la tombe - la veille de l'enterrement, il exécute le rituel de "prendre le lieu » (gazar avah), comme il désignait jadis l'emplacement du dépôt du corps dans la steppe - il s'agit d'un autre spécialiste bouddhiste que l'astrologue qui 
« ouvre la boîte dorée » (altan sav nee-) peu après le décès. En prévision des morts en hiver, des trous sont creusés au début de l'automne, avant le gel du sol, et le bon emplacement de la tombe est donc choisi en hiver parmi ces trous préalablement creusés. Dans le cas présent, la mort n'étant pas naturelle, les " porteurs d'os » ont exceptionnellement dû creuser un trou dans le sol gelé, la veille de l'enterrement.

27. Ar'jasüren et Njambuu 1992, p. 364.

28. À ne pas confondre avec la pierre [de marquage] blanche qui était posée au niveau de la tête du cadavre du temps où il était exposé (Ar'jasüren et Njambuu 1992, p. 364).

29. Marque initiale de l'alphabet créé par le moine bouddhiste Zanabazar en 1696, récupérée par le gouvernement communiste et érigée comme un symbole de la nation mongole, figurant sur le drapeau national. Des familles ajouteraient des appliqués cousus représentant ces trois mêmes éléments du Sojombo, généralement associés, dans la décoration du cercueil, au niveau des pieds (Pürev et Pürvee 2007).

30. Sur la difficulté de traduire en français des notions mongoles d'orientation de l'espace et du corps humain par les référents absolus et invariables que sont nos points cardinaux, voir Beffa et Hamayon 1983, pp. 81-111.

31. Le coton blanc sous les tissus bleu et vert peut aussi évoquer le feutre blanc placé avec la soie verte sous le corps déposé dans la steppe, auquel Badamhatan fait référence (1987, p. 302).

32. Even 1999, p. 177-178.

33. Le clergé bouddhique a répandu en Mongolie des notions particulières de souillure latente et de péché, obligeant les familles à agir chaque jour de manière faste pour "appeler [à elles] » (dallah) le bonheur (Krystyna Chabros 1992), de même qu'elles évitent d'agir de manière néfaste pour « réparer » (zasah) le malheur - ce qui ne signifie pas que les rites d'appel et de réparation résultent d'une influence bouddhique à proprement parler.

34. Plan Carpin 1965, p. 40.

35. Aussi longtemps qu'ils ont ressenti l'organisation de leur société comme reposant sur les lignages, les Mongols ne pouvaient pas adopter la notion doctrinale bouddhique de karma, puisqu'elle réfute la transmigration des âmes.

36. La renaissance de l'âme dans le corps d'un futur nouveau-né est qualifiée de bonne, et elle rejoint le principe chamanique de renaissance des âmes. Inversement, la renaissance dans le corps d'un animal est considérée comme étant mauvaise, et elle constitue une perte d'une unité de vie, entraînant à terme la non-reproduction du groupe.

37. Le salaire mensuel moyen de la famille du veuf, qui vit d'un étalage de vente de légumes, est de $20000 \varsubsetneqq(16 €)$, celui du foyer de la couturière-menuisière, tirant essentiellement sa subsistance de la vente de charbon au détail, est de 10000 ₹ $(8 €)$, celui d'un instituteur de 23000 ₹ $(19 €), 1 €$ équivalant à 1200 F.

38. Plan Carpin 1965, p. 40.

39. La " part des ancêtres » (xešig) leur était adressée par l'intermédiaire du feu allumé à côté de la tombe et les « restes de la viande sacrifiée » (bülüür) et de la «boisson de sacrifice » (sarxad) étaient distribués aux participants (Ligeti 1973, pp. 150-161).

40. Ar'jasüren et Njambuu 1992, p. 364. Laetitia Merli travaille sur les nouvelles formes de chamanismes qui apparaissent à Ulaanbaatar. Un chamane lui a dit tout le mal qu'il pense du dépôt d'une yourte miniature sur la tombe, qui capture l'âme du mort et l'empêche de s'en aller, soulevant la colère des morts (Merli 2004, p. 296).

41. Plan Carpin 1965, pp. 40-41.

42. C'est un objet rituel bouddhiste en fer qui, comme son nom l'indique, a la forme des monuments funéraires bouddhiques ou stupas.

43. Sur les gâteaux en forme de semelle comme matérialisation des empreintes des visiteurs espérés et reçus à la fête du nouvel an lunaire, voir Ruhlmann 2006, pp. 240-246, 293-297 et p. 412, et Ruhlmann 2008b. 
44. Si le nourrissement du mort dans la tombe, ou dans le cercueil, n'existe plus, il semble également que le nourrissement des mânes des ancêtres (claniques) n'existe plus. Sur la disparition de l'organisation sociale clanique aristocratique, lire les hypothèses de Rodica Pop (2002).

45. C'est communément par des gestes circulaires dirigés vers soi que, dans les temples et les foyers, on attire la fumée d'encens pour appeler [à soi] le bonheur.

46. Pour les commémorations de mort des septième, vingt-et-unième et quarante-neuvième jours, et le nourrissement de l'âme, voir Ruhlmann 2006, pp. 416-470.

47. Sur les nourritures blanches et la couleur blanche (linceul ou cercueil, fosse, nourritures pour purifier ou pour nourrir) favorisant le bon sort postmortem de l'âme, voir Ruhlmann 2006, pp. 394-470.

\section{RÉSUMÉS}

Dans une capitale provinciale de l'Est de la Mongolie, l'occasion m'a été donnée d'assister et de participer à des funérailles. Je propose d'analyser le cas particulier observé comme un exemple représentatif de la pratique mongole halh contemporaine d'enterrement des parents défunts. Cette analyse se fonde sur l'étude minutieuse du cercueil, de sa confection et de sa décoration qui requièrent un savoir-faire spécifique. Elle donne accès à la portée de cette pratique et à la conception de l'âme après la mort. Elle permet d'identifier, à travers le sens donné aux signes de la boîte et de son couvercle, des éléments de croyances chamaniques, de croyances bouddhiques et d'influences soviétiques.

In a provincial capital in eastern Mongolia, I had the opportunity to attend and take part in funeral. I propose to analyze the observed particular case as a representative example of the contemporary Halh Mongolian burial practice of the dead parents. This analysis is based on the detailed study of the coffin, its confection and its decoration requiring a specific know-how. This analysis leads to the scope of this practice and to the conception of the soul after death. Through the meaning given to the signs of the box and its lid, the analysis allows to identify the elements of shamanistic beliefs, Buddhist beliefs and soviet influences.

\section{INDEX}

Index géographique : Mongolie

Keywords : buddhism, burial, coffin, shamanism, soul, soviet atheism nomsmotscles Halh

Mots-clés : âme, athéisme soviétique, bouddhisme, cercueil, chamanisme, enterrement

\section{AUTEUR}

\section{SANDRINE RUHLMANN}

Docteur en anthropologie sociale et ethnologie. L'auteur a soutenu en 2006 à l'E.H.E.S.S. une thèse d'anthropologie sociale et ethnologie sur les pratiques alimentaires chez les Mongols Halh. Elle 
poursuit actuellement des recherches sur les techniques et les savoirs mongols dans le domaine de l'alimentation, en se concentrant sur les changements qui s'opèrent dans la capitale nationale, Ulaanbaatar. À travers l'observation et l'analyse des pratiques alimentaires, différentes conceptions relatives à la culture mongole sont étudiées, comme celles de la mort, de la naissance, de l'âme, ou encore de l'espace domestique, de l'hospitalité et du partage.sandrine_ruhlmann@yahoo.fr 